\title{
Crystal defects and spin tunneling in single crystals of $\mathrm{Mn}_{12}$ clusters
}

\author{
J.M. Hernandez, ${ }^{*}$ F. Torres, and J. Tejada \\ Departamento de Fisica Fonamental, University of Barcelona, Diagonal 647, 08028 Barcelona, Spain \\ E. Molins \\ Institut de Ciència de Materials de Barcelona (CSIC), Campus UAB, 08193 Cerdanyola, Spain
}

(Received 29 May 2002; published 30 October 2002)

\begin{abstract}
The question addressed in this paper is that of the influence of the density of dislocations on the spin tunneling in $\mathrm{Mn}_{12}$ clusters. We have determined the variation in the mosaicity of fresh and thermally treated single crystals of $\mathrm{Mn}_{12}$ by analyzing the widening of low angle x-ray diffraction peaks. It has also been well established from both isothermal magnetization and relaxation experiments that there is a broad distribution of tunneling rates which is shifted to higher rates when the density of dislocations increases.
\end{abstract}

DOI: 10.1103/PhysRevB.66.161407

PACS number(s): 75.45. $+\mathrm{j}, 75.50 . \mathrm{Xx}$

Within the last few years molecular clusters have emerged as truly interdisciplinary objects. This is so because these materials allow us to test the border between quantum and classical mechanics, ${ }^{1}$ they may also be used as a hardware for quantum computers ${ }^{2,3}$ and for low temperature magnetic cooling. ${ }^{4}$ The magnetic hysteresis in molecular clusters results from the existence of $2 S+1$ spin levels in the two wells of the magnetic anisotropy barrier. $\mathrm{Mn}_{12}$ molecular clusters have $S=10$ at low temperature and are equivalent to a single domain particle with magnetic moment $20 \mu_{B}$. The occurrence of magnetic relaxation at temperatures at which the thermal fluctuations die out is due to spin resonant tunneling between degenerate $S_{Z}$ states in the two wells of the anisotropy potential wells. ${ }^{5-15}$ To the first approximation, the spin Hamiltonian used previously ${ }^{5-15}$ to fit the magnetic data obtained for the different $\mathrm{Mn}_{12} \mathrm{Ac}$ molecular clusters is written as

$$
\mathcal{H}=-D S_{Z}^{2}+\mathcal{H}^{\prime}+\mathcal{H}_{\text {dip }}+\mathcal{H}_{\text {hf }}
$$

where $D=0.65 \mathrm{~K}^{16-18}$ and $\mathcal{H}^{\prime}$ contains anisotropy terms of fourth order of the spin operator. ${ }^{16-19}$ The last two terms correspond to the contribution of both dipolar and hyperfine fields to the transverse magnetic field. The first term of Eq. (1) generates spin levels $S_{Z}$ inside each well, while the symmetry violating terms inducing tunneling are those associated with the transverse component of the magnetic anisotropy and the transverse dipolar and hyperfine fields. Very recently, however, Chudnovsky and Garanin ${ }^{20}$ have suggested that tunneling due to the magnetoelastic coupling $\mathcal{H}_{m e}$ may be even larger than that due to the terms written in Eq. (1). This may be so, as a consequence of the local transverse anisotropy and magnetic fields associated with dislocations. Also recently, Mertes et al. ${ }^{21}$ have performed Landau-Zener relaxation studies in $\mathrm{Mn}_{12}$ clusters using different sweeping rates of the applied magnetic field. Their data clearly show that (i) there is a distribution of tunneling splittings associated with the second-order anisotropy term; (ii) the fraction of $\mathrm{Mn}_{12}$ molecules remaining in the metastable state obey the scaling law suggested theoretically for the case when the tunneling is driven by dislocations. In a series of resonant experiments at frequencies between 37 and $110 \mathrm{GHz}$, using both fresh and distorted single crystals, ${ }^{22}$ we have noticed that in order to fit the data for these last crystals, it is necessary to introduce a second-order term, $E S_{X}^{2}$. There are also electron paramagnetic resonance (EPR) data ${ }^{23}$ in which the energy separation between the $S_{Z}$ spin levels is measured. These experiments suggest that energy barrier height varies slightly from molecule to molecule, which can also be interpreted in terms of the existence of dislocations. Here we present experimental evidence of the effect of defects, mostly dislocations, on both the rate of spin tunneling and the law of relaxation in single crystals of two different $\mathrm{Mn}_{12}$ molecular clusters.

$\mathrm{Mn}_{12} \mathrm{Ac}^{24}$ and $\mathrm{Mn}_{12}$ 2Cl-benzoate, ${ }^{25,26} \mathrm{Mn}_{12} \mathrm{Cl}$, have identical magnetic cores and local symmetries, with the only difference that the easy axes of $\mathrm{Mn}_{12} \mathrm{Cl}$ lie alternatively in the direction (011) or $(0 \overline{1} 1)$, being nearly perpendicular to their nearest neighbors. That is, the spin Hamiltonian for the two type single crystals should contain the same terms. Fresh single crystals of $\mathrm{Mn}_{12} \mathrm{Ac}$ and $\mathrm{Mn}_{12} \mathrm{Cl}$ were first characterized by x-ray diffraction techniques and then we carried out the magnetic studies. The next step was to cycle the temperature of the crystals between $80 \mathrm{~K}$ and $300 \mathrm{~K}$ four times. Then we performed new $\mathrm{x}$-ray diffraction and magnetic characterization. A second rapid thermal (RT) process, similar to the first one, followed by both x-ray and magnetic characterization was also performed. During all these operations the single crystals were kept glued on the top of a glass capilar inside the measuring probe. Several runs using different fresh samples were performed for both $\mathrm{Mn}_{12} \mathrm{Ac}$ and $\mathrm{Mn}_{12} \mathrm{Cl}$.

In order to have an idea of how much a temperature change induces dislocations, we can compare the minimum stress to create a dislocation with stresses created by temperature gradients. It is easy to show that the minimum temperature change (in air) to create a dislocation in a small crystal is roughly $\Delta T_{\text {min }} \approx 3 \sigma / 2 \alpha E$, where $\sigma$ is the tensile strength, $\alpha$ is the linear expansion coefficient, and $E$ is the Young's modulus. Giving reasonable values for a molecular crystal, the minimum temperature change is about $100 \mathrm{~K}$. The RT process supposes a rapid change in the temperature of the surrounding air of the crystal of about $200 \mathrm{~K}$. When the crystal core is still at the initial temperature, the surface of the crystal starts to cool down. This large temperature 


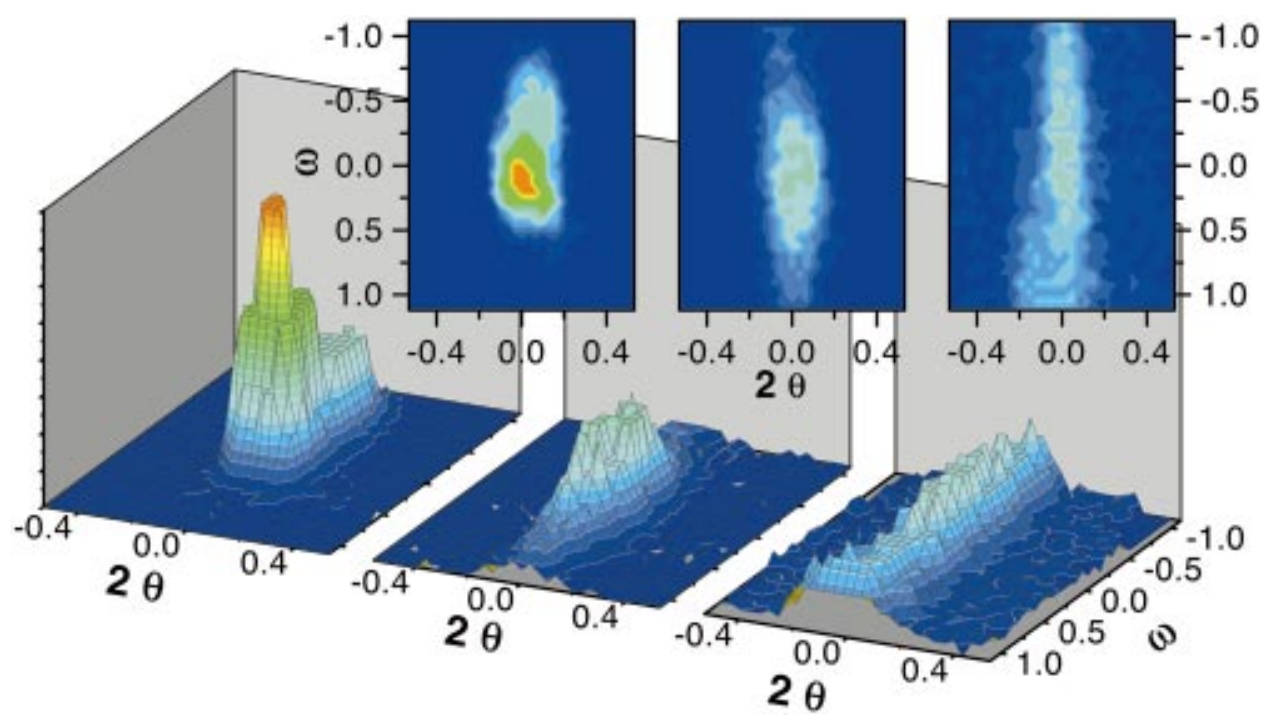

FIG. 1. (Color) $\omega-\theta$ plot of the $(\overline{2} 22)$ reflection of the $\mathrm{Mn}_{12} \mathrm{Cl}$ crystal: before any process (left), after one RT process (center), and after two RT processes (right). The insets clearly show the enlargement of the peak along $\omega$ due to the increase in mosaicity after the RT process, while keeping a constant $\theta$ width. The flattening in $\omega$ even overcomes the scan width.

gradient generates radial and tangential tensions that favor the propagation of dislocations across the crystal, probably starting at point defects frozen during the growth of the crystal and during the x-ray irradiation. The propagation of dislocations by the whole crystal converts an initial single crystal into a multidomain crystal where each element is slightly misaligned with respect to its neighbors. This is what is known as a mosaic crystal and the decrease of the average size of the domains and their misalignment is related to the widening of the diffraction peaks. As the peak widening observed in our experiments is as low as a few tenths of a degree, the samples after the thermal process are still considered as single crystals but with a larger mosaicity.

To better determine the mosaicity variation with the RT processes, ${ }^{27-29}$ we have focused our attention on low angle diffraction peaks in order to minimize the widening associated to the lack of monochromaticity (Mo $K \alpha_{1}$ and Mo $K \alpha_{2}$ ). After checking the crystal parameters and getting the crystal orientation by using an Enraf-Nonius CAD4 fourcircle $\mathrm{x}$-ray diffractometer, we choose the reflections $( \pm 2$ $\pm 2 \pm 2$ ) for comparing their shape before and after the RT process because they were low angle and intense. In Fig. 1 we show the $\omega-\theta$ plot of the ( $\overline{2} 22)$ diffraction peak, for the $\mathrm{Mn}_{12} \mathrm{Cl}$ single crystal, in its pristine form (left), after the first RT process (center) and after a second one (right). The inset clearly shows the widening of the diffraction peak along $\omega$ due to the increase of the mosaicity while the width along $2 \theta$ remains constant. It can be also seen that after the second RT process, the flattening in $\omega$ even overcomes the scan width. Assuming $^{27}$ that the average distance between dislocations is inversely related to the $\omega$ widening, and taking into account that the latter doubles approximately after each thermal treatment, see Fig. 1, it may be concluded that the number of dislocations increases near by an order of magnitude after the RT process. It is assumed that the domain size effect dominates over the effect of misalignment, otherwise the increase of the dislocation concentration would be lower. Similar results were observed in the analysis of low angle diffraction peaks for the $\mathrm{Mn}_{12} \mathrm{Ac}$ single crystal.

The magnetic characterization of the single crystals before and after the RT process include hysteresis cycles at
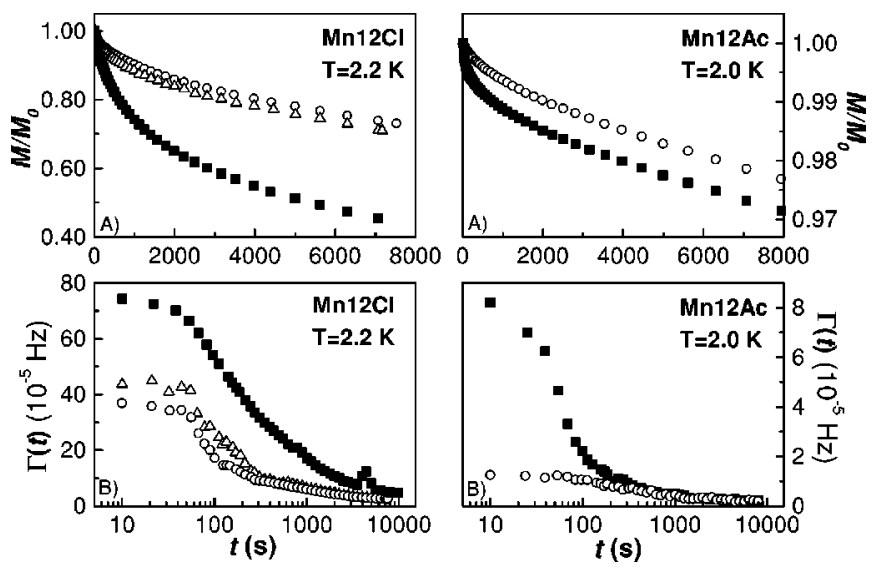

FIG. 2. (a) Magnetic relaxation of single crystals of $\mathrm{Mn}_{12} \mathrm{Ac}$ and $\mathrm{Mn}_{12} \mathrm{Cl}$ at different temperatures before (open circles) and after (open triangles, solid squares) the RT process. (b) Evolution with time of the effective relaxation rate corresponding to the data depicted in (a). The rate has been calculated as the time derivative of the logarithm of the magnetization. 


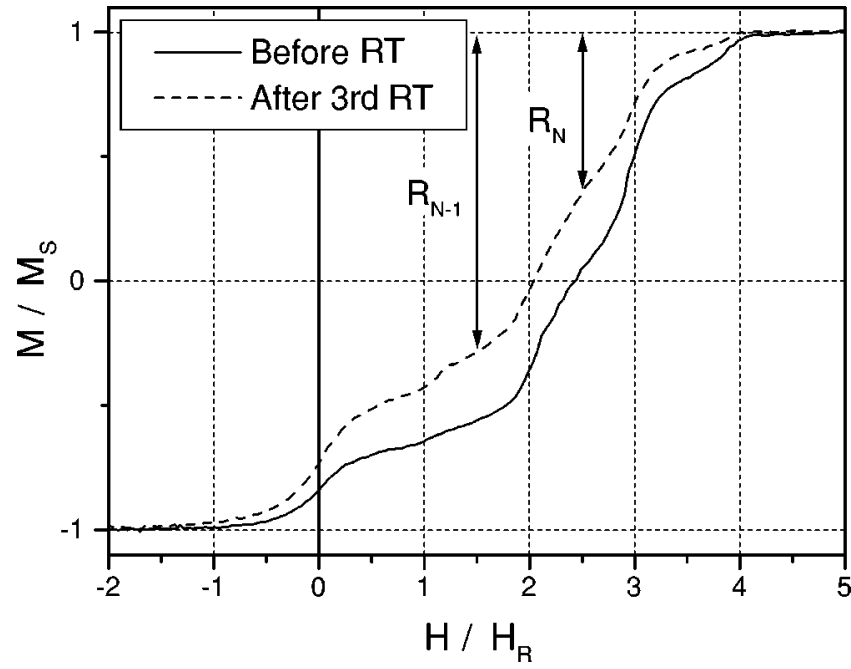

FIG. 3. Isothermal magnetization curves at $2 \mathrm{~K}$ for $\mathrm{Mn}_{12} \mathrm{Cl}$ single crystal, before (solid line) and after (dashed line) the third RT process.

different temperatures and relaxation experiments over the resonance at zero field by changing the sweeping rate of the magnetic field. The experimental evidence of changes associated to the thermal process came out from the magnetic relaxation data at zero field, see Fig. 2. In these measurements the single crystals were first placed in a field $H$ $=30 \mathrm{kOe}$ at $T=10 \mathrm{~K}$, then they were cooled until the desired temperature and the field was then switched off. The direction of the applied field coincides with the $c$ axis of the $\mathrm{Mn}_{12} \mathrm{Ac}$ crystals. In the case of the $\mathrm{Mn}_{12} \mathrm{Cl}$ single crystals, the field is applied along the easy axis direction for only half of the molecules. The data displayed in Fig. 2 are representative of those obtained for different samples of the two single crystals at different temperatures and show that both the amount of magnetization relaxing per unit time and the relaxation rate are larger after performing the RT processes.

TABLE I. Values of the tunneling probability, $\rho_{N}$, at the different resonance peaks for the $\mathrm{Mn}_{12} \mathrm{Cl}$ single crystal before, after the first, second and third thermal process deduced from hysteresis measurements recorded at different temperatures.

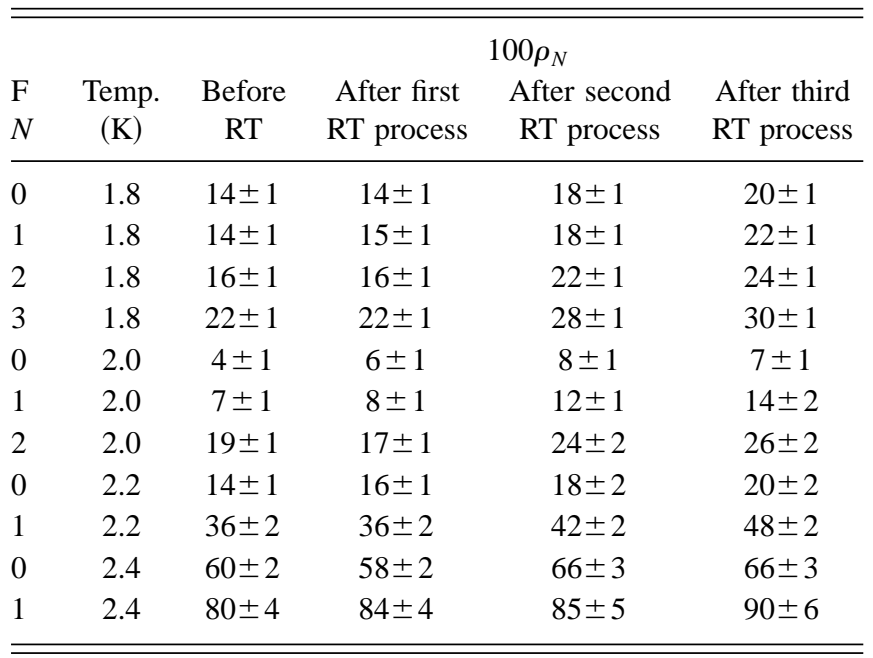

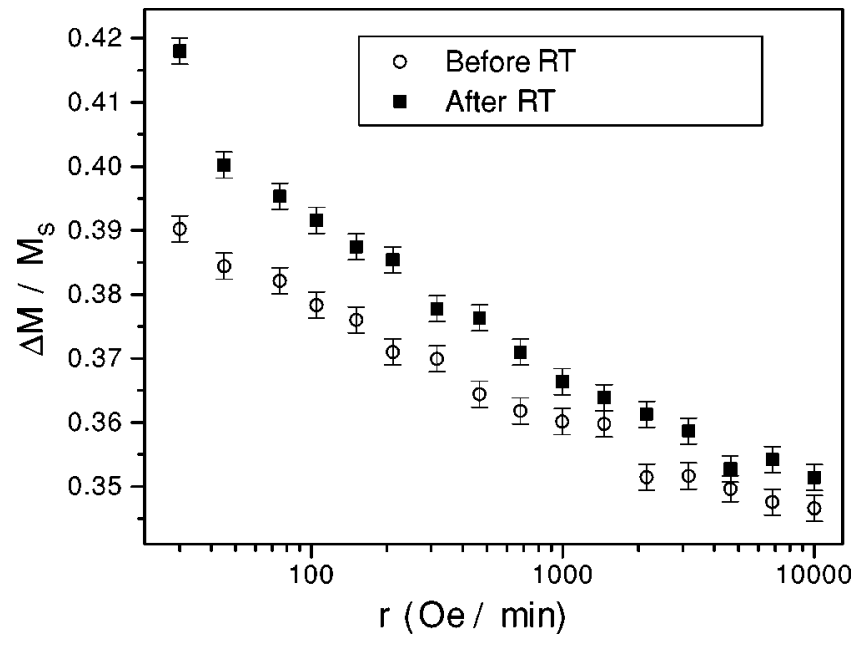

FIG. 4. Dependence on the sweeping rate of the applied magnetic field, of the variation of magnetization on a Landau-Zener experiment at $1.8 \mathrm{~K}$ and for the zero field resonance for $\mathrm{Mn}_{12} \mathrm{Ac}$ single crystal, before (solid squares) and after (open circles) the RT process.

The effect appears emphasized in Fig. 2(b), where the relaxation rate $\Gamma$ at each time is plotted as deduced from the relaxing data by using the differential exponential law $(d M$ $=-M \Gamma d t)$.

The variation of $\Gamma$ with time, see Fig. 2(b), strongly supports that the spin relaxation is a superposition of exponential decays with different rates,

$$
M(t)=M_{0} \int f(\Gamma) \exp (-\Gamma t) d \Gamma,
$$

where $M_{0}$ is the initial magnetization at $t=0$, and $\Gamma$ should correspond to the relaxation rate for the different molecules may or may not have been affected by dislocations. We have verified recently ${ }^{30}$ that the relaxation law can be fitted by a stretched exponential, its exponent depending on the concentration of dislocations. The molecules near the core of the dislocations are those for which the magnetoelastic coupling is the strongest and which, consequently, show the largest tunneling rate. This is the reason why there is a distribution of relaxation times and why the measured tunneling rate decreases with time.

In Fig. 3 we show isothermal magnetization curves at $2 \mathrm{~K}$ for the $\mathrm{Mn}_{12} \mathrm{Cl}$ single crystal before and after the RT process. It is well known that the magnetic relaxation at each resonance depends on the step number $N$, as well as on the sweep rate of the applied magnetic field. In all these experiments we have fixed this rate at $10 \mathrm{Oe} / \mathrm{s}$. In order to study the dependence of the magnetic relaxation at each resonance on the RT process, we have calculated the tunneling probability $\rho_{N}$ at each resonance, $\rho_{N}=R_{N-1}-R_{N} / R_{N-1}$, where $R_{N}$ is the fraction of molecules that remain in the metastable well after the $N$ th level crossing. In Table I we list the values of $\rho_{N}$ for the case of the $\mathrm{Mn}_{12} \mathrm{Cl}$ single crystals before and after the first, second, and third thermal process. Table I clearly shows that $\rho_{N}$ is larger for all resonances detected experimentally in the case of the single crystals suffering the RT 
process. That is, the amount of magnetization tunneling at each resonance is correlated to the crystal mosaicity detected in the x-ray experiments. In other words, the crystals having more and more dislocations show faster and faster magnetic relaxation because they have larger and larger number of molecules affected by dislocations.

The temperature tends to reduce the differences in $\rho_{N}$ for the fresh and treated crystals as it should be due to the fact that as the temperature increases we start detecting the molecules that are not affected by dislocations and are located far away from the nucleus of the dislocations.

Landau-Zener experiments have also been performed, ${ }^{21,31}$ using fresh and thermal treated single crystals of $\mathrm{Mn}_{12} \mathrm{Ac}$. The tunneling probability when one resonance is crossed at a given sweeping rate $r$ for the magnetic field depends exponentially on the tunneling splitting of the resonance. Our Landau-Zener experiments were done in the following way. First a negative saturation field was applied at the measuring temperature. Then the field was changed to $H_{1}=-2 \mathrm{kOe}$ at the highest sweeping rate and the value of magnetization was measured giving $M_{1}$. The field was immediately changed to $H_{2}=2 \mathrm{kOe}$ at a giving sweeping rate $r$, measuring $M_{2}$ after the process was finished. This procedure was repeated at different sweeping rates ranging from $30 \mathrm{Oe} / \mathrm{min}$ up to 1 $\mathrm{T} / \mathrm{min}$ and at different temperatures. The results obtained at $1.8 \mathrm{~K}$ are shown in Fig. 4. In this figure we plot the normalized variation of the magnetization $\Delta M / M_{S}=M_{2}-M_{1} / M_{S}$ as a function of $r$. One can see that this quantity increases, for all values of $r$, after the single crystals have suffered the heat treatment.

It is clear that while it is possible to perform a one-to-one correspondence between theory and experiments in the case of the ground-state tunneling for $\mathrm{Mn}_{12} \mathrm{Ac},{ }^{21}$ it is not the case in our experiments as the tunneling occurs from many different excited levels. Concerning the analysis of the data for the two types of $\mathrm{Mn}_{12}$ single crystals we have used in our experiments, we expect a qualitatively similar effect of dislocations for two reasons: dislocations introduce new terms in the spin Hamiltonian associated with both the transversal field and anisotropy, and the $\mathrm{Mn}_{12} \mathrm{Cl}$ crystals are formed by two identical blocks similar to $\mathrm{Mn}_{12} \mathrm{Ac}$ with perpendicular orientation between them. That is, following the analysis of Chudnovsky and Garanin, ${ }^{20}$ for both type of single crystals, an increase is expected in the tunneling rate as the concentration of defects increases.

Summarizing, we have shown and discussed magnetic data for different single crystals of two molecular clusters, which indicate that the existence of dislocations modify substantially the spin tunneling rate. The magnetic relaxation law is strongly affected by defects, as a consequence of the overlapping of tunneling splittings for the different spin levels.

The authors thank the EC (Grant No. IST-1999-29110) and Generalitat de Catalunya (Grant No. 2001SGR335) for financial support.
*Electronic address: jmh@ubxlab.com

${ }^{1}$ E.M. Chudnovsky, Science 274, 938 (1996); P.C.E. Stamp, Nature (London) 383, 125 (1996); B. Schwarztchild, Phys. Today 50(7), 17 (1997).

${ }^{2}$ M.N. Leuenberger and D. Loss, Nature (London) 410, 789 (2001).

${ }^{3}$ J. Tejada et al. Nanotechnology 12, 181 (2001).

${ }^{4}$ F. Torres et al. Appl. Phys. Lett. 77, 3248 (2000).

${ }^{5}$ J.R. Friedman et al. Phys. Rev. Lett. 76, 3830 (1996).

${ }^{6}$ J.M. Hernandez et al. Europhys. Lett. 35, 301 (1996).

${ }^{7}$ L. Thomas et al. Nature (London) 383, 145 (1996).

${ }^{8}$ J.A.A.J. Perenboom et al. Phys. Rev. B 58, 330 (1998).

${ }^{9}$ W. Wernsdorfer et al. Europhys. Lett. 47, 254 (1999).

${ }^{10}$ L. Bokacheva et al. Phys. Rev. Lett. 85, 4803 (2000).

${ }^{11}$ D.A. Garanin and E.M. Chudnovsky, Phys. Rev. B 56, 11102 (1997).

${ }^{12}$ F. Luis, J. Bartolomé, and J.F. Fernández, Phys. Rev. B 57, 505 (1998).

${ }^{13}$ N.V. Prokof'ev and P.C.E. Stamp, Phys. Rev. Lett. 80, 5794 (1998)

${ }^{14}$ M.N. Leuenberger and D. Loss, Europhys. Lett. 46, 692 (1999).

${ }^{15}$ A. Garg, Phys. Rev. Lett. 81, 1513 (1998).

${ }^{16}$ A.L. Barra et al., Phys. Rev. B 56, 8192 (1997).

${ }^{17}$ A.A. Mukin et al., Europhys. Lett. 44, 778 (1998).
${ }^{18}$ Y. Zhong et al., J. Appl. Phys. 85, 5636 (1999).

${ }^{19}$ I. Mirebeau et al., Phys. Rev. Lett. 83, 628 (1999).

${ }^{20}$ E.M. Chudnovsky and D.A. Garanin, Phys. Rev. Lett. 87, 187203 (2001); D.A. Garanin and E.M. Chudnovsky, Phys. Rev. B 65, 094423 (2002)

${ }^{21}$ K.M. Mertes et al., Phys. Rev. Lett. 87, 227205 (2001).

${ }^{22}$ R. Amigo et al., Phys. Rev. B 65, 172403 (2002).

${ }^{23}$ K. Park et al., cond-mat/0112172 (unpublished).

${ }^{24}$ T. Lis, Acta Crystallogr., Sect. B: Struct. Crystallogr. Cryst. Chem. 36, 2042 (1980).

${ }^{25}$ B. Albela, Ph.D. thesis, Universitat Barcelona, 1996.

${ }^{26}$ F. Luis et al. Nanotechnology 10, 86 (1999).

${ }^{27}$ R.I. Barabash, in Defect and Microstructure Analysis by Diffraction, edited by R.L. Snyder, J. Fiala, and H.J. Bunge, IUCr Monographs on Crystallography Vol. 10 (Oxford University Press, Oxford, 1999), pp. 127-140.

${ }^{28}$ A.McL. Mathieson, Acta Crystallogr., Sect. A: Cryst. Phys., Diffr., Theor. Gen. Crystallogr. 38, 378 (1982).

${ }^{29}$ A.McL. Mathieson and A.W. Stevenson, Acta Crystallogr., Sect. A: Found. Crystallogr. 41, 290 (1985); 42, 223 (1986).

${ }^{30} \mathrm{~F}$. Torres et al., cond-mat/0110538.

${ }^{31}$ L.D. Landau, Phys. Z. Sowjetunion 2, 46 (1932); C. Zener, Proc. R. Soc. London A137, 696 (1932). 